PROCEEDINGS OF THE

AMERICAN MATHEMATICAL SOCIETY

Volume 137, Number 8, August 2009, Pages 2639-2644

S 0002-9939(09)09879-7

Article electronically published on March 27, 2009

\title{
ANOTHER DICHOTOMY FOR SURFACE DIFFEOMORPHISMS
}

\author{
C. MORALES
}

(Communicated by Jane M. Hawkins)

\begin{abstract}
We prove that a $C^{1}$ generic orientation-preserving diffeomorphism of a closed orientable surface either has infinitely many periodic points with complex (nonreal) eigenvalues or is Axiom A without cycles. This improves Mañé's dichotomy.
\end{abstract}

\section{INTRODUCTION}

In this paper we prove that a $C^{1}$ generic orientation-preserving diffeomorphism of a closed orientable surface either has infinitely many periodic points with complex eigenvalues or is Axiom A without cycles. This improves Mañé's dichotomy [5], at least in the orientation-preserving case, since a generic surface diffeomorphism with infinitely many periodic points with complex eigenvalues has infinitely many attracting or repelling periodic points too. We also obtain another obstruction for the solution of Smale's Conjecture [1] concerning the $C^{1}$ genericity of Axiom A diffeomorphisms on closed surfaces, namely, the existence of infinitely many periodic points with complex eigenvalues. Let us state our result in a precise way.

Hereafter $M$ denotes a closed orientable surface, i.e., a compact connected boundaryless two-dimensional orientable Riemannian manifold. We denote by $\operatorname{Diff}^{1}(M)$ the set of $C^{1}$ diffeomorphisms $f: M \rightarrow M$ endowed with the standard $C^{1}$ topology, and by $\operatorname{Diff}_{+}^{1}(M)$ the open set of orientation-preserving elements of $\operatorname{Diff}^{1}(M)$. It turns out that $\operatorname{Diff}^{1}(M)$ is a Baire metric space. A subset $R$ of $\operatorname{Diff}^{1}(M)$ is resid$u$ al if it contains the intersection of a countable family of open and dense subsets. When we say that a $C^{1}$ generic diffeomorphism satisfies a property $(P)$ we mean that there is $R \subset \operatorname{Diff}^{1}(M)$ residual such that $(P)$ holds for every $f \in R$. The closure of a set $A$ is denoted by $\mathrm{Cl}(A)$.

By a periodic point of $f$ we mean a point $p \in M$ for which there is an integer $n \in \mathbb{N}$ such that $f^{n}(p)=p$. The minimal of such integers is called the minimal period of $p$ with respect to $f$ and is denoted by $n_{p}$ (observe that $n_{p}$ also depends on $f)$. Denote by $\operatorname{Per}(f)$ the set of periodic points of $f$. We say that $p \in \operatorname{Per}(f)$ has complex eigenvalues if the eigenvalues of the linear map $D f^{n_{p}}(p): T_{p} M \rightarrow T_{p} M$ are nonreal complex conjugated numbers.

The nonwandering set of $f, \Omega(f)$, is the set of all points $p \in M$ such that for every neighborhood $U$ of $p$ there is $n \in \mathbb{N}$ such that $f^{n}(U) \cap U \neq \emptyset$. Clearly $\Omega(f)$ is closed and $\operatorname{Per}(f) \subset \Omega(f)$.

Received by the editors August 18, 2008.

2000 Mathematics Subject Classification. Primary 37D30; Secondary 37E30.

Key words and phrases. Axiom A diffeomorphism, homoclinic tangency, complex eigenvalues. 
If $\Lambda$ is a compact invariant set of $f$, we define its stable and unstable sets by

$$
W^{s}(\Lambda)=\left\{x \in M: \omega_{f}(x) \subset \Lambda\right\} \quad \text { and } \quad W^{u}(\Lambda)=\left\{x \in M: \alpha_{f}(x) \subset \Lambda\right\}
$$

respectively. We say that $\Lambda$ is hyperbolic if there are a continuous $f$-invariant tangent bundle decomposition $T_{\Lambda} M=E_{\Lambda}^{s} \oplus E_{\Lambda}^{u}$ and constants $K, \lambda>0$ such that

$$
\left\|D f^{n}(x) / E_{x}^{s}\right\| \leq K e^{-\lambda n} \quad \text { and } \quad m\left(D f^{n}(x) / E_{x}^{u}\right) \geq K^{-1} e^{\lambda n}, \quad \forall x \in \Lambda, \forall n \in \mathbb{N},
$$

where $m(\cdot)$ is the co-norm operation. We say that $f$ is Axiom $A$ if $\Omega(f)$ is hyperbolic and $\mathrm{Cl}(\operatorname{Per}(f))=\Omega(f)$. In such a case the Spectral Decomposition Theorem [6] asserts that $\Omega(f)$ is the disjoint union of finitely many compact invariant sets $\Lambda_{1}, \cdots, \Lambda_{k}$ which are transitive and isolated. We then say that $f$ has no cycles if there is no $\left\{i_{1}, \cdots, i_{r+1}\right\} \subset\{1, \cdots, k\}$ with $i_{1}=i_{r+1}$ such that

$$
\left(W^{u}\left(\Lambda_{i_{j}}\right) \backslash \Lambda_{i_{j}}\right) \cap\left(W^{s}\left(\Lambda_{i_{j+1}}\right) \backslash \Lambda_{i_{j+1}}\right) \neq \emptyset, \quad \forall j(\bmod r) .
$$

With these definitions we can state our result.

Theorem 1.1. A $C^{1}$ generic orientation-preserving diffeomorphism of $M$ either has infinitely many periodic points with complex eigenvalues or is Axiom A without cycles.

The proof is based on Franks's Lemma and the main theorem in [7.

Let us state a corollary of Theorem 1.1. A sink of $f \in \operatorname{Diff}^{1}(M)$ is a periodic point $p$ such that the eigenvalues of $D f^{n_{p}}(p)$ have moduli less than 1. A source of $f$ is a sink of the inverse map $f^{-1}$. We denote the set of sinks (resp. sources) of $f$ by $\operatorname{Sink}(f)$ (resp. Source $(f)$ ). A dominated splitting of a nonempty compact invariant set $\Lambda$ is a continuous invariant tangent bundle decomposition $T_{\Lambda} M=E_{\Lambda} \oplus F_{\Lambda}$ with $E_{x} \neq 0$ and $F_{x} \neq 0$ for all $x \in \Lambda$ such that there are constants $K, \lambda>0$ satisfying

$$
\frac{\left\|D f^{n}(x) / E_{x}\right\|}{m\left(D f^{n}(x) / F_{x}\right)} \leq K e^{-\lambda n}, \quad \forall x \in \Lambda, \forall n \in \mathbb{N} .
$$

It was proved in 2] that for a $C^{2}$ diffeomorphism $f$ with infinitely many sinks of $M$ there is no dominated splitting for $C l(\operatorname{Sink}(f)) \backslash \operatorname{Sink}(f)$ with contracting direction, i.e., $\left\|D f^{n}(x) / E_{x}\right\| \leq K e^{-\lambda n}$ for all $x \in C l(\operatorname{Sink}(f)) \backslash \operatorname{Sink}(f)$ and $n \in \mathbb{N}$.

It is natural to ask if such a property holds if we replace $C^{2}$ by $C^{1}$ generic in its statement. The following corollary gives a partial positive answer for this question.

Corollary 1.2. For a $C^{1}$ generic orientation-preserving diffeomorphism $f$ of $M$ there is no dominated splitting for either $C l(\operatorname{Sink}(f)) \backslash \operatorname{Sink}(f)$ or $C l(\operatorname{Source}(f)) \backslash$ Source $(f)$.

\section{Proofs}

We first state Franks's Lemma, which can be found in [4, Lemma 1.1] or [7, Lemma 2.1.1].

Lemma 2.1. Let $f \in \operatorname{Diff}^{1}(M)$ and $\mathcal{W}(f) \subset \operatorname{Diff}^{1}(M)$ be a neighborhood of $f$. Then, there are $\epsilon>0$ and a neighborhhod $\mathcal{W}_{0}(f) \subset \mathcal{W}(f)$ of $f$ such that if $g^{\prime} \epsilon$ $\mathcal{W}_{0}(f),\left\{x_{1}, \cdots, x_{n}\right\} \subset M$ is a finite set, $U \subset M$ is a neighborhood of $\left\{x_{1}, \cdots, x_{n}\right\}$ and $L_{i}: T_{x_{i}} M \rightarrow T_{g^{\prime}\left(x_{i}\right)} M$ are linear maps satisfying $\left\|L_{i}-D g^{\prime}\left(x_{i}\right)\right\|<\epsilon(\forall i=$ $1, \cdots, n)$, then there is $g \in \mathcal{W}(f)$ such that $g(x)=g^{\prime}(x)$ in $\left\{x_{1}, \cdots, x_{n}\right\} \cup(M \backslash U)$ and $D g\left(x_{i}\right)=L_{i}$ for every $i=1, \cdots, n$. 
We use this lemma to prove the following proposition. Given $f \in \operatorname{Diff}(M)$ we denote by Saddle $(f)$ the set of all $p \in \operatorname{Per}(f)$ such that $D f^{n_{p}}(p)$ has real eigenvalues $\lambda_{p}, \sigma_{p}$ with $\left|\lambda_{p}\right|<1<\left|\sigma_{p}\right|$. Denote by $E_{p}^{s}$ and $E_{p}^{u}$ the eigenspaces corresponding to $\lambda_{p}$ and $\sigma_{p}$ respectively. Denote by $\angle(E, F)$ the angle between the linear subspaces $E, F \subset T_{p} M, p \in M$.

Proposition 2.2. There is a residual subset $\mathcal{R}_{+} \subset \operatorname{Diff}_{+}^{1}(M)$ such that if $f \in \mathcal{R}_{+}$ has finitely many periodic points with complex eigenvalues, then there are $\gamma>0$ and a neighborhood $\mathcal{U}(f) \subset \operatorname{Diff}_{+}^{1}(M)$ of $f$ such that $\angle\left(E_{p}^{s}, E_{p}^{u}\right)>\gamma$ for all $g \in \mathcal{U}(f)$ and $p \in \operatorname{Saddle}(g)$.

Proof. Given $f \in \operatorname{Diff}^{1}(M)$ we denote by $\operatorname{Spir}(f)$ the set of $p \in \operatorname{Per}(f)$ with complex eigenvalues. Denote by $2_{c}^{M}$ the set formed by the compact subsets of $M$ endowed with the Hausdorff topology. Define the set-valued map

$$
\begin{aligned}
& \Phi: f \in \operatorname{Diff}^{1}(M) \rightarrow 2_{c}^{M}, \\
& \Phi(h)=\operatorname{Cl}(\operatorname{Spir}(h)) .
\end{aligned}
$$

Clearly $\Phi$ is lower-semicontinuous, so the set

$$
\mathcal{R}=\left\{f \in \operatorname{Diff}^{1}(M): \Phi \text { is semicontinuous at } f\right\}
$$

is residual in $\operatorname{Diff}^{1}(M)$. Then, by the Kupka-Smale Theorem [6], we can assume that every periodic point $p \in \operatorname{Per}(f)$ of $f \in \mathcal{R}$ is hyperbolic; namely, the linear map $D f^{n_{p}}(p)$ has no eigenvalues of modulus 1. Define

$$
\mathcal{R}_{+}=\mathcal{R} \cap \operatorname{Diff}_{+}^{1}(M) .
$$

It is clear that $\mathcal{R}_{+}$is residual in $\operatorname{Diff}_{+}^{1}(M)$.

Now suppose that $f \in \mathcal{R}_{+}$has finitely many periodic points with complex eigenvalues. As each element of $\operatorname{Per}(f)$ is hyperbolic we have that $\operatorname{Spir}(f)$ consists of finitely many sinks or sources. Then, there are neighborhoods $\mathcal{U}_{0}(f)$ of $f$ and $U_{0}$ of $\operatorname{Spir}(f)$ such that

$$
\operatorname{Per}(g) \cap U_{0} \subset \operatorname{Spir}(g), \quad \forall g \in \mathcal{U}_{0}(f) .
$$

Since $\operatorname{Diff}_{+}^{1}(M)$ is open and $f \in \operatorname{Diff}_{+}^{1}(M)$ we can assume that $\mathcal{U}_{0}(f) \subset \operatorname{Diff}_{+}^{1}(M)$. But $\Phi$ is semicontinuous at $f$. So, we have the following remark.

Remark 2.3. We can assume that $C l(\operatorname{Spir}(g)) \subset U_{0}$ for all $g \in \mathcal{U}_{0}(f)$.

Let $\epsilon>0$ and $\mathcal{W}_{0}(f) \subset \mathcal{W}(f)$ be the constant and the neighborhood (respectively) given by Lemma 2.1 applied to $\mathcal{W}(f)=\mathcal{U}_{0}(f)$. We claim that $\mathcal{U}(f)=\mathcal{W}_{0}(f)$ works.

Suppose by contradiction that this is not true. Then, there is a sequence $g_{m} \in \mathcal{W}_{0}(f)$ such that for all $m$ there is $p_{m} \in \operatorname{Saddle}\left(g_{m}\right)$ such that $\alpha_{m}=$ $\angle\left(E_{p_{m}}^{s}, E_{p_{m}}^{u}\right) \rightarrow 0$ as $m \rightarrow \infty$. Note that $D g_{m}^{n_{p_{m}}}\left(p_{m}\right) \in G L_{+}(2, \mathbb{R})$ for all $m$ since $g_{m} \in \operatorname{Diff}_{+}^{1}(M)$. Then, by Lemma 3.2, p. 384 in [3], there is $t_{m} \in[-1,1]$ such that $T_{t_{m} \alpha_{m}} \circ D g_{m}^{n_{p_{m}}}\left(p_{m}\right)$ has a complex (nonreal) eigenvalue ( $T_{\alpha}$ denotes the rotation of angle $\alpha$ ). Hence the eigenvalues of $T_{t_{m} \alpha_{m}} \circ D g_{m}^{n_{p_{m}}}\left(p_{m}\right)$ are nonreal complex conjugated numbers.

Since $\alpha_{m} \rightarrow 0$ and $t_{m} \in[-1,1]$ we have that $t_{m} \alpha_{m} \rightarrow 0$ too. Then, we can fix $m$ large so that

$$
\left\|T_{t_{m} \alpha_{m}} \circ D g_{m}^{n_{p_{m}}}\left(p_{m}\right)-D g^{n_{p_{m}}}\left(p_{m}\right)\right\|<\epsilon .
$$


To simplify we write $T=T_{t_{m} \alpha_{m}}, g^{\prime}=g_{m}, p=p_{m}$, and so on. Then, $g^{\prime} \in \mathcal{U}_{0}(f)$ and $p \in \operatorname{Saddle}\left(g^{\prime}\right)$, so $p \notin U_{0}$ by (11).

Set $x_{i}=g^{i-1}(p)$ for $i=1, \cdots, n$ and define the linear operators $L_{i}: T_{x_{i}} M \rightarrow$ $T_{g^{\prime}\left(x_{i}\right)} M$ by

$$
L_{i}= \begin{cases}D g^{\prime}\left(x_{i}\right), & i=1, \cdots, n-1, \\ T \circ D g^{\prime}\left(x_{n}\right), & i=n .\end{cases}
$$

By (22) we have that $\left\|L_{i}-D g^{\prime}\left(x_{i}\right)\right\|<\epsilon, \forall i=1, \cdots, n$. Then, by Lemma 2.1 there is $g \in \mathcal{U}_{0}(f)$ such that $g(x)=g^{\prime}(x)$ in $\left\{x_{1}, \cdots, x_{n}\right\} \cup(M \backslash U)$, and $D g\left(x_{i}\right)=L_{i}$ for every $i=i, \cdots, n$. Then, $p \in \operatorname{Per}(g)$ has period $n_{p}$ and by the chain rule we have $D g^{n_{p}}(p)=T \circ D\left(g^{\prime}\right)^{n_{p}}(p)$. Hence the eigenvalues of $D g^{n_{p}}(p)$ are nonreal complex conjugated numbers too. This implies that $p \in \operatorname{Spir}(g)$, which contradicts Remark 2.3 since $p \notin U_{0}$. This contradiction finishes the proof.

We use this proposition to prove the following one.

Proposition 2.4. If $\mathcal{R}_{+}$is the residual subset of $\operatorname{Diff}_{1}^{+}(M)$ in Proposition 2.2. then for all $f \in \mathcal{R}^{+}$with finitely many periodic points with complex eigenvalues there is an open and dense subset $\mathcal{A}(f)$ of $\mathcal{U}(f)$ such that every $g \in \mathcal{A}(f)$ is Axiom A without cycles.

Proof. Notice that no $g \in \mathcal{U}(f)$ has a homoclinic tangency, i.e., a nontransversal intersection between the stable and unstable manifolds of some hyperbolic periodic point. Indeed, if such a tangency exists, then we could unfold it to obtain $g^{\prime}$ close to $g$ (thus $g^{\prime} \in \mathcal{U}(f)$ ) exhibiting $p^{\prime} \in \operatorname{Saddle}\left(g^{\prime}\right)$ such that $\angle\left(E_{p}^{s}, E_{p}^{u}\right)<\gamma$, contradicting Proposition 2.2. Then, by the main theorem in 7], there is a dense subset $\mathcal{A}(f) \subset \mathcal{U}(f)$ all of whose elements are Axiom A without cycles (see p. 966 of 77$)$. Then, $\mathcal{A}(f)$ is also open since the set of Axiom A diffeomorphisms without cycles is (e.g. [6]). This concludes the proof.

Next we prove two topological lemmas which seem to be standard (their proofs are included for the sake of completeness). Recall that $C l(\cdot)$ denotes the closure operation. For simplicity we say that a subset $A$ of a metric space $X$ is open-dense in $X$ if it is simultaneously open and dense in $X$.

Lemma 2.5. Let $X$ be a metric space and $A, B, C \subset X$. If $A$ is open-dense in $X$, $B$ is open in $X$ and $C \subset B$ is open-dense in $B$, then $(A \backslash C l(B)) \cup C$ is open-dense in $X$.

Proof. It is clear that $(A \backslash C l(B)) \cup C$ is open, so we only need to prove that it is dense. For this choose $x \in X$. Since $A$ is dense there is a sequence $x_{n} \in A$ converging to $x$. If $x_{n} \notin C l(B)$ for all $n$ large, we have that $x_{n} \in(A \backslash C l(B)) \cup C$ for all $n$ large; thus $x$ is a cluster point of $(A \backslash C l(B)) \cup C$. Otherwise we can assume that $x_{n} \in C l(B)$ for all $n$. Since $C$ is dense in $B$ we can choose for all $n$ a point $x_{n}^{\prime} \in C$ close to $x_{n}$. Thus the sequence $x_{n}^{\prime}$ also converges to $x$ and so $x \in C l(C)$. So, $x$ is a cluster point of $(A \backslash C l(B)) \cup C$. Since $x$ is arbitrary we obtain the proof.

We use this lemma to prove the following.

Proposition 2.6. Let $R$ be a residual subset of $\operatorname{Diff}_{+}^{1}(M)$. Suppose that there is a disjoint union $R=A \cup O$ where $O$ is open in $\operatorname{Diff}_{+}^{1}(M)$. If $O^{\prime} \subset O$ is open-dense in $O$, then $A \cup O^{\prime}$ is residual in $\operatorname{Diff}_{+}^{1}(M)$. 
Proof. As $A \cap O=\emptyset$ and $R=A \cup O$ we have $A=R \backslash O$. Then, $A \cup O^{\prime}=(R \backslash O) \cup O^{\prime}$. But $R$ is residual, so we can write

$$
R=\bigcap_{n} O_{n}
$$

where each $O_{n}$ is open-dense in $\operatorname{Diff}_{+}^{1}(M)$. Then, $R \backslash O=\bigcap_{n}\left(O_{n} \backslash O\right)$ and so

$$
A \cup O^{\prime}=(R \backslash O) \cup O^{\prime}=\bigcap_{n}\left[\left(O_{n} \backslash O\right) \cup O^{\prime}\right] .
$$

Now, notice that each $\left(O_{n} \backslash C l(O)\right) \cup O^{\prime}$ is open-dense in $\operatorname{Diff}_{+}^{1}(M)(\forall n)$ by Lemma 2.5 applied to $X=\operatorname{Diff}_{+}^{1}(M), A=O_{n}, B=O$ and $C=O^{\prime}$. Then,

$$
\bigcap_{n}\left[\left(O_{n} \backslash C l(O)\right) \cup O^{\prime}\right]
$$

is residual in $\operatorname{Diff}_{+}^{1}(M)$. But

$$
\bigcap_{n}\left[\left(O_{n} \backslash O\right) \cup O^{\prime}\right] \supset \bigcap_{n}\left[\left(O_{n} \backslash C l(O)\right) \cup O^{\prime}\right],
$$

so

$$
\bigcap_{n}\left[\left(O_{n} \backslash O\right) \cup O^{\prime}\right]
$$

is residual in $\operatorname{Diff}_{+}^{1}(M)$. Then, $A \cup O^{\prime}$ also is by (3). This finishes the proof.

Proof of Theorem 1.1, Let $R_{+}$be the residual subset of $\operatorname{Diff}_{+}^{1}(M)$ given in Proposition 2.2 Define

$$
A=\left\{f \in \mathcal{R}^{+}: f \text { has infinitely many periodic points with complex eigenvalues }\right\} .
$$

If $f \in R_{+}$has finitely many periodic points with complex eigenvalues, we consider the neighborhood $\mathcal{U}(f)$ of $f$ given in that proposition. This allows us to define the open set $O$ as the union of all such neighborhoods. Define $R=A \cup O$. It follows that $R_{+} \subset R$, so $R$ is residual in $\operatorname{Diff}_{+}^{1}(M)$. Moreover, $A \cap O=\emptyset$ by Remark 2.3 .

Now using Proposition 2.4 we can define $O^{\prime}$ as the union of all $\mathcal{A}(f)$ such that $f \in \mathcal{R}_{+}$has finitely many complex eigenvalues. It follows from this proposition that $O^{\prime} \subset O$ is open-dense in $O$. Then, $A \cup O^{\prime}$ is residual in $\operatorname{Diff}_{+}^{1}(M)$ by Proposition 2.6. It follows from Proposition 2.4 (and the definition of $A$ ) that any element of $A \cup O^{\prime}$ satisfies one of the alternatives in Theorem 1.1. This completes the proof.

Proof of Corollary 1.2. Suppose by contradiction that for a $C^{1}$ generic diffeomorphism $f$ of $M$ both $C l(\operatorname{Sink}(f)) \backslash \operatorname{Sink}(f)$ and $C l(\operatorname{Source}(f)) \backslash \operatorname{Source}(f)$ have a dominated splitting.

Since $f$ is generic we have the inclusion

$$
C l(\operatorname{Spir}(f)) \backslash \operatorname{Spir}(f) \subset(C l(\operatorname{Sink}(f)) \backslash \operatorname{Sink}(f)) \cup(C l(\operatorname{Source}(f)) \backslash \operatorname{Source}(f)) .
$$

It follows that $C l(\operatorname{Spir}(f)) \backslash \operatorname{Spir}(f)$ has a dominated splitting (since $C l(\operatorname{Sink}(f)) \backslash$ $\operatorname{Sink}(f)$ and $C l$ (Source $(f)) \backslash$ Source $(f)$ do), which is a contradiction unless $f$ has finitely many periodic points with complex eigenvalues. Then, $f$ is Axiom A without cycles by Theorem 1.1 and so both $\operatorname{Sink}(f)$ and Source $(f)$ are finite sets. Then, both $C l(\operatorname{Sink}(f)) \backslash \operatorname{Sink}(f)$ and $C l(\operatorname{Source}(f)) \backslash \operatorname{Source}(f)$ must be empty, which is a contradiction once more. This proves the result. 


\section{ACKNOWLEDGMENTS}

The author would like to thank Professor E. R. Pujals, who, in 1999, pointed out a mistake in a preliminary version of this paper. He also thanks Professor M. J. Pacifico for useful conversations. The author was partially supported by CNPq, FAPERJ and PRONEX-Brazil.

\section{REFERENCES}

[1] Abdenur, F., Bonatti, C., Crovisier, S., Diaz, L. J., Generic diffeomorphisms on compact surfaces. Fund. Math. 187 (2005), no. 2, 127-159. MR2214876 (2006m:37024)

[2] Asaoka, M., Markov covers and finiteness of periodic attractors for diffeomorphisms with a dominated splitting. Ergodic Theory Dynam. Systems 20 (2000), no. 1, 1-14. MR1747033 (2001b:37040)

[3] Bonatti, C., Diaz, L. J., Pujals, E. R., A $C^{1}$-generic dichotomy for diffeomorphisms: Weak forms of hyperbolicity or infinitely many sinks or sources. Ann. of Math. (2) 158 (2003), no. 2, 355-418. MR2018925 (2007k:37032)

[4] Franks. F., Necessary conditions for stability of diffeomorphisms. Trans. Amer. Math. Soc. 158 (1971), 301-308. MR0283812(44:1042)

[5] Mañé, R., An ergodic closing lemma. Ann. of Math. (2) 116 (1982), 503-540. MR678479 (84f:58070)

[6] Newhouse, S., Lectures on Dynamical Systems. In Dynamical Systems, Progress in Mathematics (CIME Lectures 1978), 8, pages 1-114. Birkhäuser, Boston, 1980. MR.589590 (81m:58028)

[7] Pujals, E. R., Sambarino, M., Homoclinic tangencies and hyperbolicity for surface diffeomorphisms. Ann. of Math. (2) 151 (2000), 961-1023. MR1779562 (2001m:37057)

Instituto de Matemática, Universidade Federal do Rio de Janeiro, C. P. 68.530, 21945-970, Rio DE Janeiro, RJ, BraziL

E-mail address: morales@impa.br 